\title{
Embolisation of large pulmonary artery pseudoaneurysm with conservative treatment of delayed coll extrusion
}

Singapore Med J 2020; 61(3): 162-164 https://doi.org/10.11622/smedj.2020031

Dear Sir,

We herein describe the case of a 59-year-old Chinese man with a history of Stage IV squamous cell carcinoma of the lung, which was recently diagnosed in January 2017. He was referred to our department after presenting with massive haemoptysis (140 mL). At the time, his oxygen saturation was 94\%, heart rate 136 beats per minute, blood pressure 97/59 mmHg and haemoglobin level $8.4 \mathrm{~g} / \mathrm{dL}$. Computed tomography (CT) pulmonary angiography showed a pulmonary artery pseudoaneurysm (PAP) of the left upper lobe pulmonary artery, which measured $1.9 \mathrm{~cm} \times 1.4 \mathrm{~cm} \times 1.7 \mathrm{~cm}$ and was encased by the large left upper lobe mass (Fig. 1).

The patient then underwent balloon-assisted coiling of the pseudoaneurysm and the left upper lobe pulmonary artery. A $8 \mathrm{~mm}$ $\times 4 \mathrm{~cm}$ balloon (Mustang ${ }^{\mathrm{TM}}$; Boston Scientific, Natick, MA, USA) was inflated across the left pulmonary artery bifurcation via an 8-French (Fr) long sheath (BRITE TIPTM; Cordis, Hialeah, FL, USA). The aneurysm was subsequently packed with one $14 \mathrm{~mm} \times$ $60 \mathrm{~cm}$ and two $16 \mathrm{~mm} \times 60 \mathrm{~cm}$ detachable coils (Ruby®; Penumbra Inc, Alameda, CA, USA), introduced by a 2.7-Fr microcatheter (PROGREAT®; Terumo, Tokyo, Japan) via the same 8-Fr sheath (Figs. 2a \& b). The post-embolisation angiogram showed no filling of the pseudoaneurysm (Fig. 2c), although the neck of the pseudoaneurysm was still visualised. However, as there was a risk of prolapse of the additional coils into the pulmonary artery, the decision was made to stop. Following the procedure, the patient's haemoptysis reduced significantly to $<5 \mathrm{~mL}$ /day.

However, the haemoptysis recurred eight days later. Repeat CT pulmonary angiography demonstrated flow and an interval increase in the size of the left upper lobe PAP, now measuring $2.3 \mathrm{~cm} \times 2.2 \mathrm{~cm} \times 2.0 \mathrm{~cm}$ (Figs. 3a \& b). An attempt was made to deploy a $14 \mathrm{~mm} \times 59 \mathrm{~mm}$ balloon-expandable stent (BeGraft; Bentley, Hechingen, Germany) from the main to the lower lobe pulmonary artery to exclude the PAP, which was dilated to $16 \mathrm{~mm}$. The attempt was not successful due to stent retraction and poor coverage. We felt that the tapering nature, lack of stenosis, wide calibre, and distensibility of the pulmonary artery led to the unsuccessful attempt. We then supplemented this with a direct injection of $1 \mathrm{~mL}$ of $50 \%$ glue (Histoacryl $\mathbb{R}$; B Braun, Melsungen, Germany) and lipiodol (Ultrafluide; Guerbet, Villepinte, France) mixture under ultrasonography guidance. While there was initially no flow in the PAP, ultrasonography performed the next day showed persistent flow within the PAP (Fig. 3c). At that point, the haemoptysis had ceased and the patient was managed with combined radiotherapy and immunotherapy with pembrolizumab.
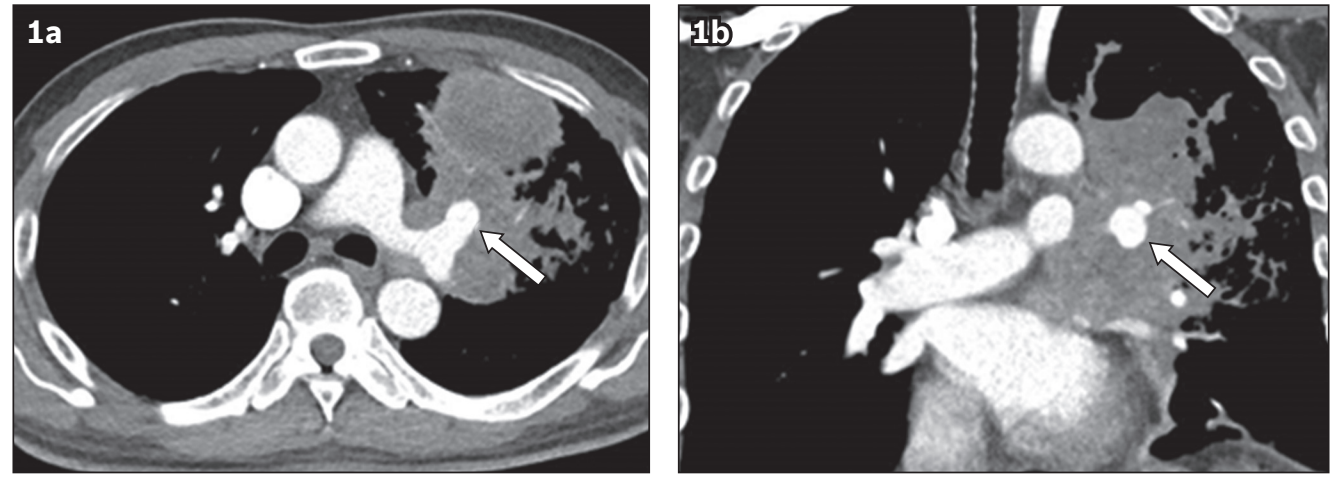

Fig. 1 (a) Contrast-enhanced axial CT pulmonary angiogram shows the left upper lobe pulmonary artery pseudoaneurysm (arrow) encased by the left upper lobe mass. (b) Coronal image shows the height of the pseudoaneurysm (arrow).
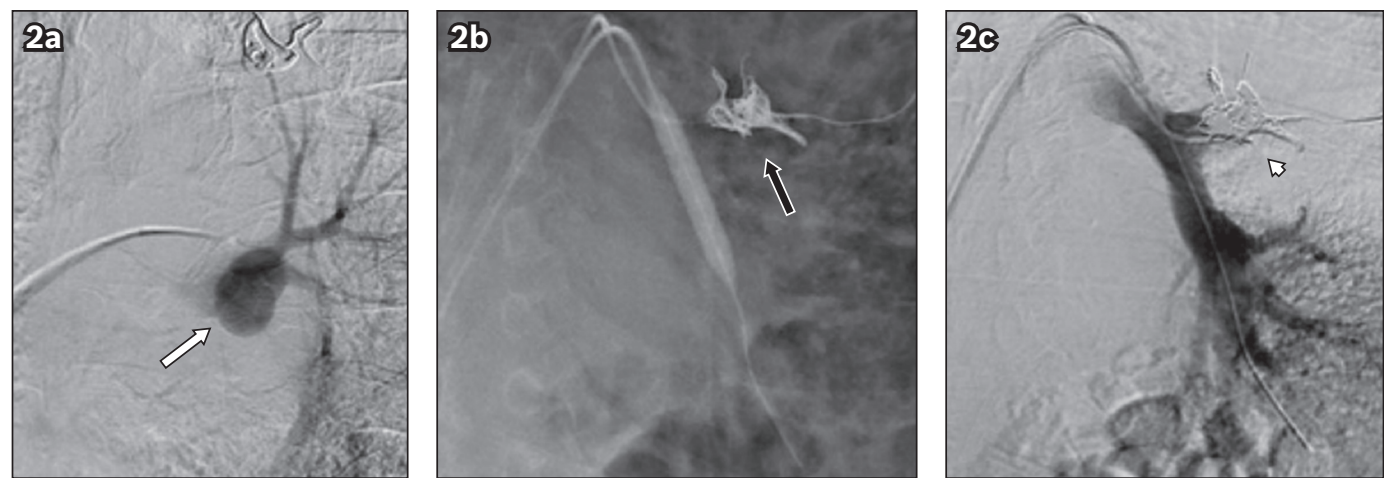

Fig. 2 Pulmonary artery angiogram shows (a) the left upper lobe pulmonary artery pseudoaneurysm (arrow) and (b) the deployment of Ruby coils with the assistance of an 8-mm balloon (black arrow). (c) Post-embolisation digital subtraction angiogram shows no flow into the left upper lobe pulmonary artery (arrowhead). 

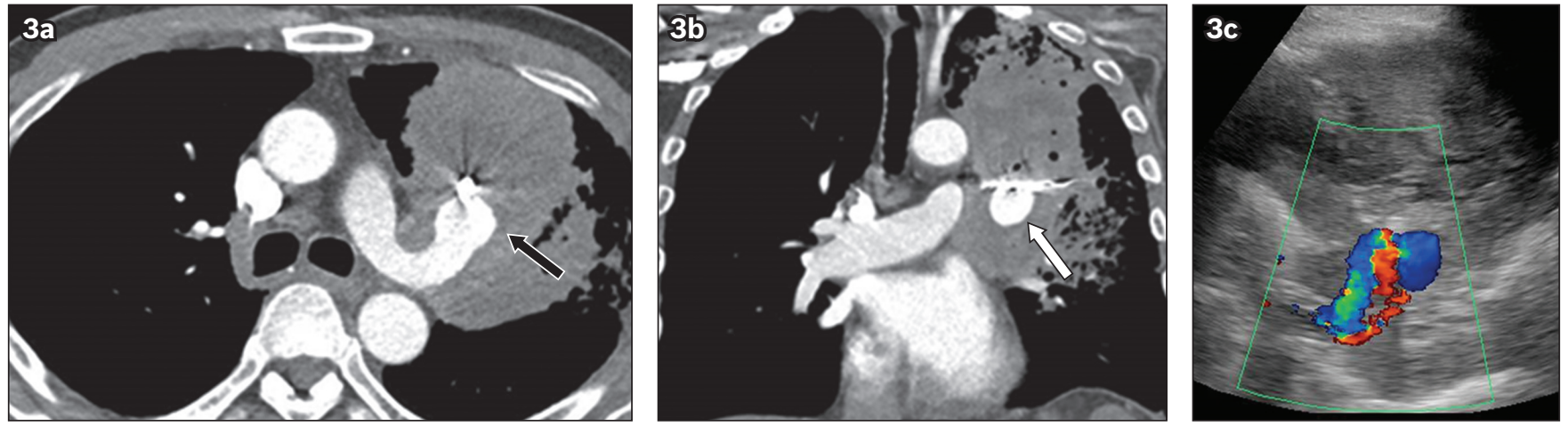

Fig. 3 (a) Contrast-enhanced axial CT pulmonary angiogram shows an interval increase in size of the left upper lobe pulmonary artery pseudoaneurysm (black arrow). (b) Coronal CT pulmonary angiogram shows the interval increase in height of the pseudoaneurysm (white arrow). (c) Transthoracic Doppler US image shows persistent flow in the pulmonary pseudoaneurysm.
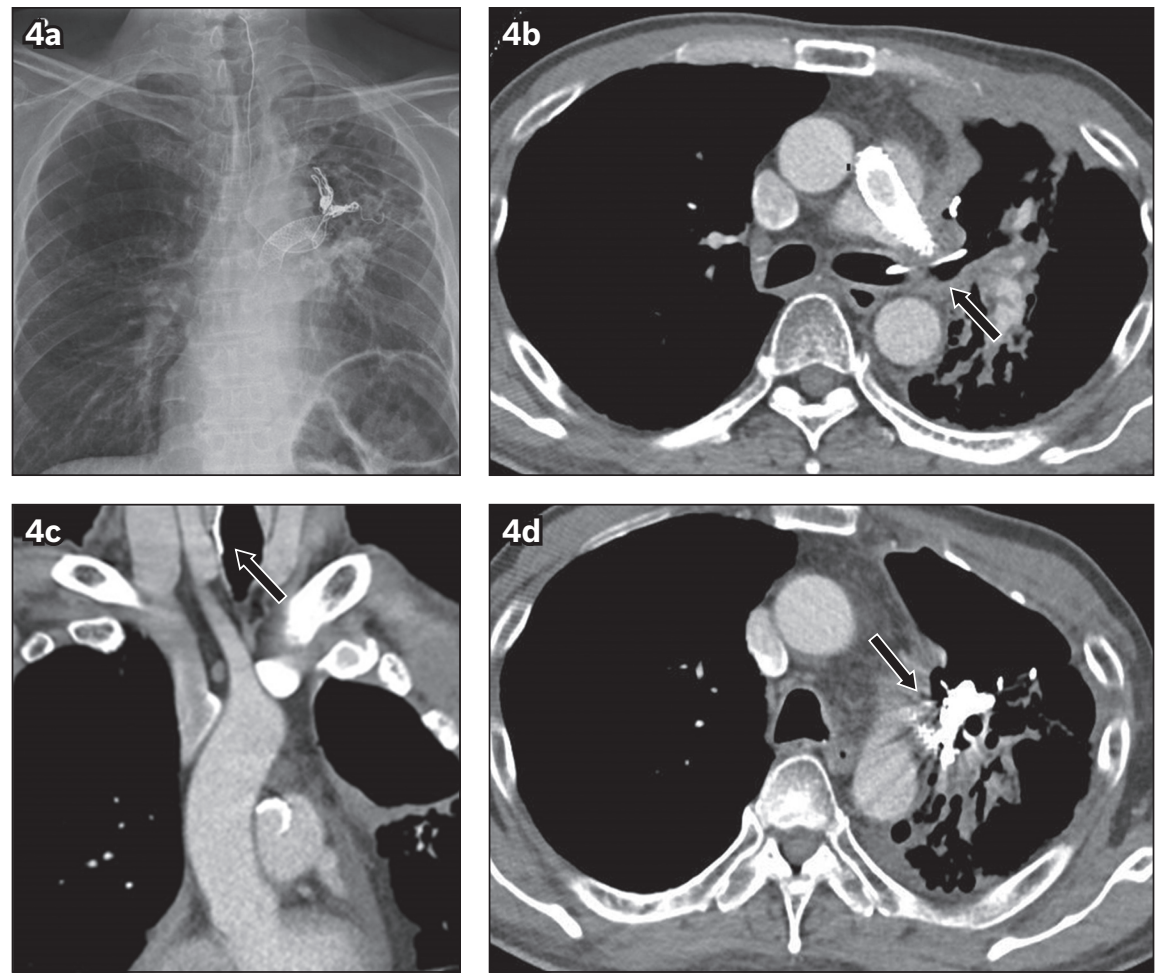

Fig. 4 (a) Chest radiograph shows migration of an uncoiled portion of the embolisation coil superiorly into the neck. (b) Contrast-enhanced axial CT image shows protrusion of the embolisation coil into the left main bronchus (arrow). (c) Coronal CT image shows that the upper margin of the coil is located in the trachea but the tip cannot be visualised in this study (arrow). (d) Axial image shows that the pulmonary artery pseudoaneurysm is partially thrombosed(arrow)

Nine months later, the patient presented with fever, cough and blood-stained sputum. Chest radiography showed further retraction of the stent and migration of a coil up the trachea, indicating fistulisation of the coil through the tumour and into the bronchus (Fig. 4a). This was confirmed on CT (Figs. 4b \& c), which also showed that the PAP had thrombosed (Fig. 4d).

While bronchoscopic retrieval was an option, we elected to manage this conservatively, as the patient remained asymptomatic apart from a mild globus sensation. Lateral neck radiography performed the following day showed that the coil had been swallowed and was in the oesophagus (Fig. 5a). Subsequent radiographs showed that the coil moved through the bowel and was eventually passed out (Figs. 5b \& c).

PAP can lead to potentially life-threatening haemoptysis, especially when it occurs within a consolidated area of lung where the potential for tissue breakdown is high, and can lead to uncontrolled rupture. Swift and accurate diagnosis is crucial because the mortality rate associated with a ruptured PAP can be as high as 50\%. ${ }^{(1)}$ The prevalence of PAPs ranges from 5\% to $11 \%$ in those presenting with haemoptysis and who underwent either bronchial or pulmonary angiography, or both. ${ }^{(2)}$ To our knowledge, only five previous reports have been published on spontaneous PAPs arising within primary lung neoplasms, with four of these five cases involving squamous cell carcinomas. ${ }^{(3-7)}$

Various embolisation techniques for PAPs have been described in the literature. They usually involve either coil embolisation of the arterial branch supplying the aneurysmal $\operatorname{sac}^{(8)}$ or filling of the sac itself with coils. ${ }^{(9)}$ Embolisation has also been accomplished 

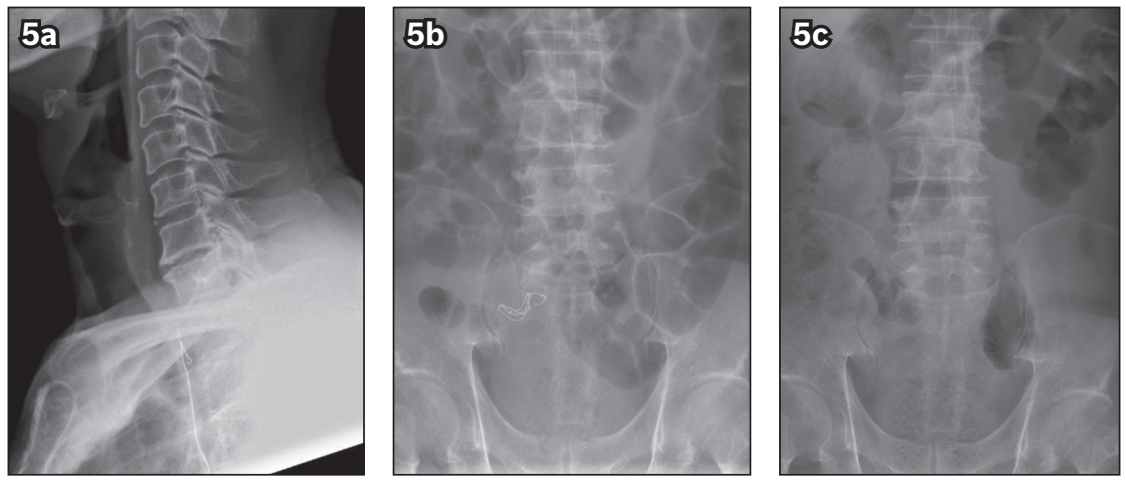

Fig. 5 (a) Lateral neck radiograph shows migration of the coil from the trachea into the upper oesophagus. (b) Abdominal radiograph taken the following day shows interval migration of the coil from the upper oesophagus into the lower abdomen. (c) Abdominal radiograph taken seven days later confirms that the coil is no longer visualised.

with detachable silicone balloons, gelfoam, polyvinyl alcohol particles, N-butyl cyanoacrylate glue ${ }^{(10)}$ and plug embolisation devices. ${ }^{(11)}$ Reports of the use of covered stents are rare and, to our knowledge, none have been described for a PAP. ${ }^{(12)}$ Ultimately, the choice of embolisation technique used depends on the site, type and anatomy of the pseudoaneurysm and also the comfort, ability and experience of the interventionist. Embolisation-related complications in the context of pseudoaneurysms are infrequently encountered and mainly consist of coil migration, damage to the vessel wall and delayed bacterial contamination of the thrombus. ${ }^{(13)}$ In the literature, there were only two reported cases of coil extrusion following embolisation procedures involving the pulmonary arteries, and both patients underwent a lobectomy..$^{(14,15)}$

Considering that a pseudoaneurysm does not possess a true intact wall, any interventional procedure for pseudoaneurysm repair is at risk of primary failure, especially if it involves coil deployment, which may easily penetrate the weak wall. Our case report demonstrates the difficulty of treating PAPs. It is our view that deploying a covered stent in this region may yield unexpected results due to the aforementioned reasons, and this patient may have benefited from embolisation of the entire left pulmonary artery after the initial failure. Secondly, we demonstrated that procedure-related complications such as coil migration may be managed conservatively if the coil is extruded via the aerodigestive tract, as long as the haemoptysis does not recur and the patient remains hemodynamically stable. Finding the most suitable embolisation agent that is both effective in treating a pseudoaneurysm and has a low risk of complications remains a challenge.

Yours sincerely,

\section{Sakktivel Elangovan ${ }^{1}$, Chow Wei $\underline{\underline{T o o}}^{1}$}

${ }^{1}$ Department of Vascular and Interventional Radiology, Singapore General Hospital, Singapore. too.chow.wei@singhealth.com.sg

\section{References}

1. Chen Y, Gilman MD, Humphrey KL, et al. Pulmonary artery pseudoaneurysms: clinical features and CT findings. AJR Am J Roentgenol 2017; 208:84-91.

2. Sbano H, Mitchell AW, Ind PW, Jackson JE. Peripheral pulmonary artery pseudoaneurysms and massive hemoptysis. AJR Am J Roentgenol 2005; 184:1253-9.

3. Akpinar E, Turkbey B, Canyigit $\mathrm{M}$, et al. Bleeding pulmonary artery pseudoaneurysm secondary to squamous cell lung cancer: computed tomography findings and endovascular management. Acta Radiol 2006; 47:944-6.

4. Padrones SS, Lisbona RL, Gratacos AR, Rodriguez AN, Diaz-Jimenez AP. Pulmonary artery pseudoaneurysm arising from a lung tumor. J Bronchology Interv Pulmonol 2009; 16:274-6.

5. Oliver TB, Stevenson AJ, Gillespie IN. Pulmonary artery pseudoaneurysm due to bronchial carcinoma. Br J Radiol 1997; 70:950-1.

6. Kim SY, Kim HR, Song JS, et al. Pseudoaneurysm due to squamous cell carcinoma of the lung: two cases of spontaneous resolution after chemotherapy. Cancer Res Treat 2009; 41:237-40.

7. Gomez-Jorge J, Mitchell SE. Embolization of a pulmonary artery pseudoaneurysm due to squamous cell carcinoma of the lung. J Vasc Interv Radiol 1999; 10:1127-30.

8. Remy J, Smith M, Lemaitre L, Marache P, Fournier E. Treatment of massive hemoptysis by occlusion of a Rasmussen aneurysm. AJR Am J Roentgenol 1980; 135:605-6.

9. Davidoff AB, Udoff EJ, Schonfeld SA. Intraaneurysmal embolization of a pulmonary artery aneurysm for control of hemoptysis. AJR Am J Roentgenol 1984; $142: 1019-20$.

10. Markowitz DM, Hughes SH, Shaw C, et al. Transcatheter detachable balloon embolotherapy for catheter-induced pulmonary artery pseudoaneurysm. J Thorac Imaging $1991 ; 6: 75-8$.

11. Chellamuthu S, Bottomley JR. Treatment of a large pulmonary artery pseudoaneurysm secondary to fungal infection using Amplatzer plugs: new embolisation devices for the management of haemoptysis. Respir Med Case Rep 2012; 5:78-80.

12. Weinstock BS, Haim YD. Pulmonary artery stenting in a patient with Takayasu's arteritis using a novel balloon-expandable covered stent. SAGE Open Med Case Rep 2019; 7:2050313X1984195.

13. Bean SH, Sadler DJ, Gray RR, So CB, Morris D. Embolization of pulmonary pseudoaneurysms secondary to metastatic synovial sarcoma of the right atrium. J Vasc Interv Radiol 1999; 10:649-52.

14. Abad J, Villar R, Parga G, et al. Bronchial migration of pulmonary arterial coil. Cardiovasc Intervent Radiol 1990; 13:345-6.

15. Elhusseiny M, Moawad A, AbdAlla D, Amer T. Pulmonary artery coil: unexpected expectorated foreign body. Chest 2016; 149(Suppl):A421. 